but difficulties arise in activating the fragment for further use. This is illustrated in a semisynthesis of human $\beta$-MSH by Burton and Lande (J. Am. chem. Soc., 92, 3746; 1970), utilising the porcine hormone as an intermediate. Human $\beta$-MSH possesses four more amino acids than porcine $\beta$-MSH and a tetrapeptide was therefore synthesised with the intention of extending the $\mathrm{NH}_{2}$-terminus of the porcine molecule. Unfortunately porcine $\beta$-MSH has two $\mathrm{NH}_{2}$ groups, the $\alpha-\mathrm{NH}_{2}$ group at position 1 and the $\varepsilon-\mathrm{NH}_{2}$ group at position 6 , and the coupling reaction led to the formation of products with the tetrapeptide attached at more than one site. Inevitably the yield of the required $\alpha$-linked hormone was low. In semisynthesis it is clearly important that, before coupling, all reactive side chains should be protected to ensure that only terminal residues are free to participate in the formation of peptide bonds. Herein lies the difficulty.

Offord (Nature, 221, 37; 1969) has reported that carboxyl groups in peptides can be protected under mild conditions by reaction with phenyldiazomethane. This forms benzyl esters at the side chains of all aspartic and glutamic acid residues but it also esterifies the C-terminal carboxyl group; the latter, however, can be regenerated selectively by the action of an esterase. When the fragment is initially obtained from a natural polypeptide by the action of trypsin, the same enzyme can be used to remove the C-terminal benzyl group. Similarly $\mathrm{NH}_{2}$ groups can be reversibly blocked in a precursor polypeptide before the required fragment is released by proteolytic cleavage. Provided the peptide was initially present within the sequence of the precursor and not at the $\mathrm{NH}_{2}$-terminus, the enzymically released fragment will have a free $\mathrm{NH}_{2}$-group at the $\mathrm{N}$-terminus; the $\varepsilon-\mathrm{NH}_{2}$ groups remain protected. By these reactions, one fragment can be prepared with only a C-terminal carboxyl group and another with only an N-terminal $\mathrm{NH}_{2}$ group, appropriate to the coupling reaction. In this way, linking of the first peptide to the second can be made to take place specifically between the Cterminus of the one and the N-terminus of the other. While these reactions seem straightforward, it is true to say that few reports have appeared in the literature on the preparation of new polypeptides by the combination of natural fragments.

A semisynthesis of a different type has involved the preparation of an insulin in which the B1 phenylalanine was replaced by iodophenylalanine, with retention of biological activity (Offord, Nature, 227, 718; 1970). To perform the conversion, the $\mathrm{Al}$ and B29 $\mathrm{NH}_{2}$ groups were protected by trifluoracetylation and the remaining $\mathrm{NH}_{2}$ group at $\mathrm{B} 1$ was blocked by reaction with phenylisothiocyanate. The B1 phenylalanine residue was removed by treatment with trifluoracetic acid, giving rise to a fragment of insulin which lacked only the B1 residue. Coupling of iodophenylalanine to the insulin fragment was easily achieved. Obviously the semisynthesis of this homogeneous derivative is more practicable than a total synthesis of the specifically iodinated insulin from the constituent amino acids.

Dyckes, Creighton and Sheppard (Nature, 247, 202; 1974) have described an intriguing semisynthesis of an analogue of trypsin inhibitor. The 58-residue polypeptide was cleaved specifically at a methionylarginine residue by cyanogen bromide, which converted the methionine residue to homoserine lactone and conferred weak acylating properties on the fragment. A peptide bond then formed spontaneously between the homoserine residue and the adjacent arginine residue, restoring the intact polypeptide chain. The product, which carried a residue of homoserine in place of methionine, retained the inhibitory action of the natural molecule. Corradin and Harbury (Biochem. biophys. Res. Commun., 61, $1400 ; 1974)$ performed a similar experiment with cytochrome $c$, which has no stabilising disulphide bridges. They found that cyanogen bromide cleavage gave rise to two fragments which could reunite; again the homoserine analogue retained biological activity.

In the general case, cleavage of a polypeptide at methionine would not be accompanied by a tendency for the fragments to recombine. Only in situations where the three-dimensional structure of the cleaved molecule is the same or very similar to that of the natural molecule will the newly released termini be held in juxtaposition for intramolecular condensation.

These are early days in the development of semisynthetic procedures. Future applications will be awaited with interest.

\title{
Between molecules and collision complexes
}

from our Chemical Physics Correspondent

IT is almost three years (Nature, 240, 257; 1972) since I drew attention to the identification of (HF) and expressed the hope that $\mathrm{H}_{2} \mathrm{OHF}$ would also be identified in the gas phase. This has now been done bj Bevan, Legon, Millen and Rogers (J. Chem. Soc. Commun., page 341; 1975) who find a clear-cut microwave spectrum near $14 \mathrm{GHz}$ and $29 \mathrm{GHz}$.

It is the hydrogen of the HF which forms the hydrogen bond; the $\mathrm{O} \ldots \mathrm{F}$ distance is $268 \mathrm{pm}$ and the dipole moment is $12.8 \times 10^{-30}$ C m (3.82 debye). There is an intensity alternation associated with the spin statistics of the two equivalent $\mathrm{H}$ attached to the oxygen, but it is not clear if the whole molecule is planar (point group $\mathrm{C}_{2 \mathrm{v}}$ ) or whether the fluorine lies out of the $\mathrm{H}_{2} \mathrm{O}$ plane (point group $\mathrm{C}_{\mathrm{s}}$ ). As expected the intensity seems to be proportional to the product of the partial pressures of each component and with an optimum total pressure of $100 \mathrm{~Pa}$ the lines are necessarily broad and such clear identification must have required the use of much experimental skill.

But though hydrogen bonds have been known for some time and such gas phase molecules have long been postulated, the extension of this work to the charge transfer species $\left(\mathrm{CH}_{3}\right)_{3}$ N.ICF $\mathrm{CF}_{3}$ is even more remark- able (Legon, Millen and Rogers, J. Chem. Soc. Chem. Commun., page $580 ; 1975)$ even though it is based on earlier infrared evidence (Mishra and Pullin, Aust. J. Chem., 24, 2493; 1971). For this species, which is a symmetric top, lines for $J$ from 30 to 37 span $26 \mathrm{GHz}$ to $33 \mathrm{GHz}$ and are identified. With only one value of $B$ for such a large molecule the structural information is not very precise, but suggests an N . . . I distance of $293.2 \mathrm{pm}$. Habit sets the expression 'N ....I' with dots suggesting a non-bonded distance, but really the charge transfer complex has now a clear-cut identity and perhaps a full bond, based on charge transfer, should be shown.

And also unexpected is the spectroscopic detection, this time with molecular beam apparatus, by Harris, Janda, Novick and Klemperer (J. Chem. Phys., 63, 881; 1975), of the rare gas species Ar.OCS. Both radiofrequency and microwave transitions were seen and they establish the structure as basically $\mathrm{T}$-shaped with the argon adjacent to the carbon at a distance of $358 \mathrm{pm}$, slightly greater than the van der Waals' radius sum.

All of these molecules with selfrespecting structures, lifetimes and dipole moments fill in the gap between true molecules and sticky collision complexes. 